\title{
TRANSFORMATION ARTISANALE DE LA VIANDE DE PORC À RODRIGUES : PRATIQUES DE FABRICATION ET QUALITÉ DES PRODUITS
}

\author{
TRADITIONAL PROCESSING OF PORK MEAT IN RODRIGUES ISLAND: \\ PROCESSING TECHNIQUES AND PRODUCT QUALITY
}

\section{Procesamiento tradicional de Carne de Cerdo en Rodrígues: TÉCNICAS DE PROCESAMIENTO Y CALIDAD DEL PRODUCTO}

\author{
K. Boodhoo ${ }^{1 *}$ C. Lisette ${ }^{2}$ S.J. Santchurn ${ }^{2}$
}

\begin{abstract}
Mots-clés : Viande porcine - Transformation des produits alimentaires - Qualité des aliments - Maurice.
\end{abstract}

Keywords: Pork - Food processing - Food quality Mauritius.

Palabras clave: Carne de cerdo - Procesamiento de alimentos - Calidad de los alimentos - Mauricio.

\begin{abstract}
'île Rodrigues est connue pour ses produits traditionnels Lfabriqués à base de porc. Cependant, et contrairement à la production agricole y compris l'élevage porcin $(1,2,3)$, le secteur de la transformation de la viande porcine a été peu étudié. Cette étude a été menée pour préciser les caractéristiques du secteur de la transformation de la viande de porc, notamment les profils socio-économiques des transformateurs, les types de produits fabriqués, les matières premières, ingrédients et additifs utilisés, les techniques et matériel de fabrication, ainsi que les modes de stockage et de conditionnement des produits finis. Une évaluation du niveau de conformité des unités de fabrication a également été effectuée au regard de la réglementation mauricienne sur l'hygiène alimentaire.
\end{abstract}

Une enquête auprès de tous les transformateurs de porc recensés $(n=57)$ a été réalisée de décembre 2010 à janvier 2011 à l'aide d'un questionnaire et d'une liste de contrôle. Excepté pour deux unités semi-industrielles, le secteur est constitué de petites unités de fabrication de charcuterie, opérant majoritairement à temps partiel (96 p.100), et gérées principalement par des hommes (65 p.100). Les principaux produits fabriqués sont la saucisse chinoise, le jambon rodriguais et le boudin noir (figure 1). Le kitouz, le boucané, le rôti de porc, le porc salé, la galantine, la saucisse créole et le bacon rodriguais sont également produits mais surtout pour des occasions festives. Les techniques de fabrication les plus utilisées sont le salage et le séchage, la cuisson n'étant employée que pour certains produits, comme le rôti de

\footnotetext{
1. Département des productions et systèmes agricoles, faculté d'agriculture, université de Maurice, Réduit, île Maurice.

2. Département des sciences agricoles et alimentaires, faculté d'agriculture, université de Maurice, Réduit, île Maurice.

* Auteur pour la correspondance

E-mail : k.boodhoo@uom.ac.mu
}
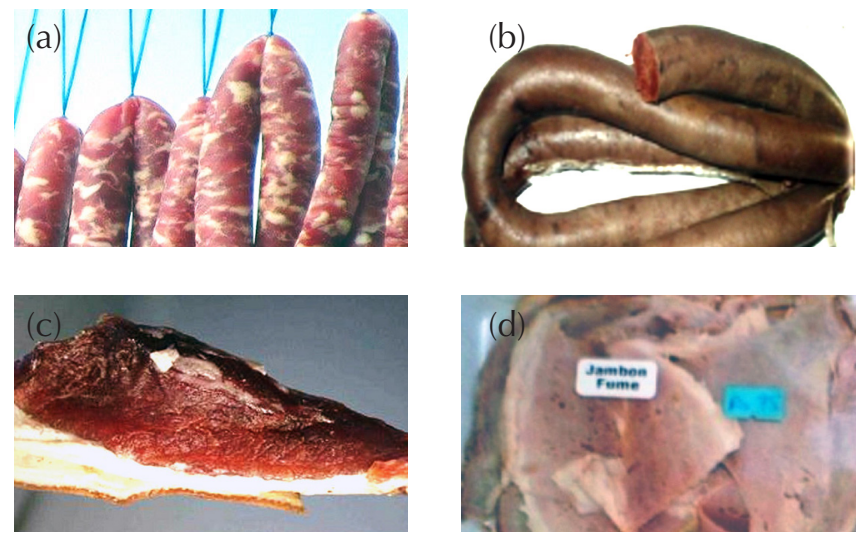

Figure 1 : exemples de produits traditionnels à base de viande de porc à l'île Rodrigues : (a) saucisse chinoise, (b) boudin noir, (c) kitouz et (d) jambon fumé.

porc et le boudin noir. La viande de porc provient d'élevages locaux, alors que les ingrédients comme les épices, le sel et le sucre sont importés de l'île Maurice.

Tous les artisans charcutiers recensés $(n=57)$ fabriquaient leurs produits dans la cuisine familiale à l'aide de matériel de faible coût, et en adoptant une technologie rudimentaire et empirique, et des recettes traditionnelles. La plupart des transformateurs ne suivaient pas les codes de bonnes pratiques de fabrication et étaient bien en-deçà des normes d'hygiène requises par la réglementation mauricienne. Quatre-vingts pour cent d'entre eux n'avaient pas de système de contrôle de leur procédé de fabrication. Ainsi, par exemple dans la fabrication des saucisses chinoises, le degré de hachage de la viande pouvait varier d'un hachage grossier à très fin. Les quantités d'ingrédients étaient mesurées de façon approximative en utilisant des cuillères et des tasses. Parmi ces artisans, 88 p.100 n'étiquetaient pas leurs produits après l'emballage. Par ailleurs, en début de la chaîne de fabrication, I'abattage du porc s'effectuait souvent dans des conditions insalubres augmentant ainsi les risques de contamination microbiologiques de la carcasse. La plupart des artisans (95 p.100) stockait la viande fraîche à température ambiante, favorisant ainsi la multiplication des germes. Pour ces diverses raisons, les produits finis étaient clairement hétérogènes au niveau des dimensions, de la teneur en gras, de la couleur, de l'aspect général et de la durée de conservation. 
En conclusion, les fabricants devront mettre en œuvre les bonnes pratiques de fabrication et d'hygiène de base, afin d'assurer une qualité standard des produits finis et d'améliorer leur qualité sanitaire. Une étude ultérieure sera réalisée pour mieux définir la qualité microbiologique des produits.

\section{BIBLIOGRAPHIE}

. BELMIN R., PORPHYRE V., 2013. Les indications géographiques (IG) : outils de conquête du marché de masse pour les territoires ruraux défavorisé des pays du Sud. Le cas du porc de l'île Rodrigues. In : VI
Congrès international SYAL, Les systèmes agroalimentaires localisés face aux opportunités et défis du nouveau contexte global, Florianópolis, Brésil, 22-25 mai 2013.

2. CSO, 2010. Digest of statistics on Rodrigues. Mauritius, Central Statistical Office. www.gov.mu/portal/goc/cso/file/rod10.pdf (accessed 12 Oct. 2010)

3. NEPAD/FAO, 2005. Strengthening the agro processing capacity of Rodrigues. Investment report. Rome, FAO, Investment Centre Division. ftp.fao.org/docrep/fao/008/ae961e/ae961e00.pdf (accessed 15 Dec. 2011)

Accepted 30 April 2015; Online publication June 2015 